\title{
Estudando o Comportamento dos Preços do Etanol no Estado de São Paulo: Persistência e Volatilidade como um Processo de Longa Memória
}

\section{Studying the Behavior of Ethanol Prices in São Paulo: Persistence and Volatility as a Long Memory Process}

\author{
Cleiton Roberto da Fonseca Silva* \\ Sinézio Fernandes Maia**
}

\begin{abstract}
Resumo: Esse artigo estudou a volatilidade dos preços do etanol hidratado no estado de São Paulo durante o período 2000/2011. A aplicação de modelos da família $\mathrm{ARCH}$, bem como a consideração da série de preços do etanol como um processo de longa memória tanto na média quanto na variância condicional permitiu estimar o grau de persistência aos choques promovidos na volatilidade dos preços desse produto. A análise geral legitima a preocupação do governo com o alto grau de persistência na volatilidade e incentiva o aprofundamento da discussão em torno da formação de estoques reguladores. Os resultados corroboram ainda a teoria defendida pelo Ipea (2010) segundo a qual a consolidação dos carros flex-fuel ajudou a promover a estabilidade dos preços e indica que, embora o período pós-crise seja caracterizado por uma maior persistência da volatilidade, o patamar apresentado nesse período é inferior aquele evidenciado antes de 2004, quando os carros flex-fuel não promoviam impacto no mercado.
\end{abstract}

Palavras-chave: Etanol. Volatilidade. Commodities.

Abstract: This article studied the volatility of hydrated ethanol in São Paulo for $2000 / 2011$ period. The application of $\mathrm{ARCH}$ models, as well as the consideration of price series of ethanol as a process of a long memory in both the mean and conditional variance allowed to estimate the degree of persistence in volatility shocks. The analysis supports the government's concern with the high degree of persistence in volatility, and encourages the depth of the discussion around formation of buffer stocks. The results corroborate the theory advocated by Ipea (2010) that the consolidation of flex-fuel cars helped to foster price stability and indicates that the post crisis is characterized by a higher persistence of volatility; the level shown this period is lower than before 2004, when the flex-fuel cars did not encourage market impacts.

\footnotetext{
Mestre em Economia pelo Programa de Pós-Graduação em Economia (PPGE) da Universidade Federal da Paraíba (UFPB). E-mail: cleiton.roberto@yahoo.com.br

* * Professor do Programa de Pós-Graduação em Economia (PPGE) da Universidade Federal da Paraíba (UFPB).E-mail: sinezio@ccsa.ufpb.br
} 
Keywords: Ethanol. Volatility. Commodities.

JEL Classification: C22; Q11; Q42.

\section{Introdução}

A preocupação com o gerenciamento de riscos é algo que caracteriza uma série financeira. Não por acaso a volatilidade, variação de uma série de interesse em torno de uma média, é um ponto relevante estudado pelos economistas. Em geral, nos estudos empíricos, o ponto chave está em obter parâmetros que facilitem o gerenciamento do risco, a definição de estratégias ótimas de investimento e a aplicação de políticas públicas adequadas.

No mercado financeiro esse tipo de estudo é bastante difundido, visando especialmente os dois primeiros objetivos mencionados. $\mathrm{O}$ estudo sobre volatilidade permite aplicação em diversas nuances. Recentemente, na área da economia agrícola, os trabalhos são direcionados às commodities internacionais. Trata-se de um tema de interesse, especialmente se considerado o impacto que alterações no preço desses produtos podem exercer sobre a taxa de câmbio, balanço de pagamentos, inflação e demais variáveis macroeconômicas de países como o Brasil, caracterizados como grande exportador de commodities agrícolas.

De fato, o Brasil é o maior produtor de cana-de-açúcar no mercado internacional. Desse deriva o etanol, produto que ganhou espaço nas últimas décadas em virtude, entre outros motivos, de promover um processo mais "limpo" de energia a um custo relativamente baixo. Países como Estados Unidos, Índia, China e Japão entraram nesse mercado, derivando o etanol de produtos diferenciados como milho e beterraba, cientes do potencial que o mercado pode oferecer. No Brasil, existe um esforço permanente para a colocação definitiva do etanol como commodity internacional, uma vez que essa consolidação geraria grandes benefícios à economia nacional.

Internamente, as discussões sobre o etanol devem ser colocadas considerando-se eventos cronologicamente importantes. Em meio aos incentivos promovidos pelo Programa Nacional do Álcool (Proálcool) na década de 1970 até o boom que os carros flex-fuel promoveram no consumo e nos preços praticados interna-mente, uma preocupação se tornou pertinente nos últimos anos: o movimento dos preços. A volatilidade atingida pelos preços do etanol motivou preocupações e o debate sobre quais medidas devem ser adotadas para:

a) diminuir a volatilidade dos preços;

b) impedir que, frente aos diversos choques a que tais preços estão submetidos, o nível de preço do etanol flutue por muito tempo fora do equilíbrio. 
De fato, desde 2009 o governo planeja estimular a formação de estoques reguladores do produto como uma forma de auxiliar a manutenção do nível de preços. No mais, no final desse mesmo ano, instituíram-se os agentes de comercialização como ente intermediário entre o produtor e as distribuidoras. Essa proposta visava diminuir a volatilidade com a presença de novos agentes e incentivar a estocagem do produto.

Apesar de tais preocupações, os estudos sobre a volatilidade dos preços do etanol são bastante escassos na literatura, mesmo que a discussão sobre as demais commodities em geral tenha um espaço consolidado. Recentemente, o Ipea (2010) divulgou relatório onde sugere que o boom dos carros flex-fuel ocorrido em 2004 e as medidas prudenciais utilizadas para estimular a formação de estoques promoveram uma queda na volatilidade dos preços do produto e em sua persistência nos últimos anos. Nesse sentido, a proposta desse artigo é estudar a dinâmica da volatilidade dos preços do etanol, procurando determinar se a preo-cupação com tal volatilidade é pertinente e se as colocações defendidas pelo Ipea (2010) passam pelo teste empírico.

Para isso, serão considerados tanto modelos básicos de volatilidade empregados na literatura, como aqueles da família $\mathrm{ARCH}$, quanto o modelo fracionário ARFIMA-FIGARCH na tentativa de, se for o caso, respeitar o processo gerador dos dados e captar a persistência de longo prazo assumido pela série de preços do etanol. A estratégia inclui a divisão dos dados em períodos específicos de interesse, visando identificar a dinâmica da volatilidade em momentos distintos. O estudo é importante uma vez que analisar a volatilidade dos preços do produto e identificar seus níveis de persistência auxilia a formulação de políticas públicas para manutenção do equilíbrio de preços, que tem se tornado centro do debate sobre biocombustíveis no Brasil em período recente.

Para atingir tais objetivos, o artigo está dividido da seguinte maneira: a próxima seção apresenta uma revisão sobre as estratégias adotadas e os resultados obtidos pelos estudos de volatilidade aplicados às commodities agrícolas, discutindo principalmente informações pertinentes sobre o etanol e os eventos particulares de interesse. Em seguida, discutem-se os dados e os modelos de volatilidade que serão aplicados: os modelos básicos da família ARCH (GARCH, EGARCH e TARCH) e o modelo ARFIMA-FIGARCH. A quarta seção finalmente discute os resultados. No mais, seguem as conclusões, referências e apêndice.

\section{Análise de Volatilidade Aplicada às Commodities Agrícolas e ao Etanol}

A análise de volatilidade é um tema bastante aplicado ao mercado de commodities. Como exemplo internacional recente, é possível citar o trabalho de Tadesse e Guttormsen (2011), que analisam o comportamento dos preços das 
commodities na Etiópia com o objetivo de identificar estratégias de estabilização de preços e de intervenção governamental. Em termos nacionais, um estudo bastante completo foi realizado por Teixeira et al. (2008). Os autores analisam, através de modelos da família $\mathrm{ARCH}$, o comportamento do retorno do cacau, do boi gordo e do café, importantes commodities nacionais. Os resultados sugerem um alto grau de persistência da volatilidade com efeitos assimétricos em todas as séries. No caso do boi gordo e do cacau foi possível identificar ainda a presença do efeito alavancagem. Ou seja, choques negativos impactando mais fortemente a série em relação a choques positivos.

Arêdes e Pereira (2008) aplicaram modelos ARIMA e SARIMA ao preço do trigo no estado do Paraná e evidenciaram a presença de heterocedasticidade condicional nos resíduos, corroborando a utilização de modelos da família ARCH. Os resultados sugerem que os modelos $\mathrm{ARCH}$ e TARCH apresentaram as melhores performances no tocante às previsões pontuais. Adicionalmente, a série de preços do trigo apresenta volatilidade persistente a choques. Mais: essa volatilidade é regida por efeitos assimétricos, embora não sejam de alavancagem. Resultado idêntico foi encontrado por Nicola et al. (2008) ao analisar a volatilidade dos preços do açúcar no mercado futuro brasileiro. Ou seja: uma grande persistência da volatilidade, indicando que os choques se dissipam lentamente. No entanto, nesse caso, não se encontrou evidências de assimetria.

No tocante à cana-de-açúcar, matéria prima do etanol, Cunha et al. (2009) utilizaram modelos GARCH e evidenciaram que não há persistência na volatilidade do preço da cana-de-açúcar recebido pelo produtor paulista. Resultado diverso é encontrado por Campos e Piacenti (2007). Os autores aplicam modelos de heterocedasticidade condicional nas séries de retornos mensais da soja, mamona e cana-de-açúcar no período de 1967 a 2006. Os resultados indicam grande persistência para todos eles. Mais: a cana-de-açúcar é o produto com maior volatilidade e com maior persistência. Em suma, a cana-de-açúcar e a mamona apresentam variância condicional assimétrica, com a presença de efeito alavancagem em relação à primeira.

Nota-se que a análise da volatilidade aplicada às commodities em geral tem espaço consolidado na literatura. O objetivo desse artigo, conforme comentado, é focar um produto cuja discussão é incipiente e que está prestes a se tornar commodity internacional: o etanol ${ }^{1}$. Preocupações ambientais relacionadas aos combustíveis fósseis e a constituição de um novo componente para a matriz energética estimularam a utilização o etanol em países como Japão, Estados Unidos, China e Índia, que formam o grupo dos principais concorrentes brasileiros².

$1 \quad$ O foco se dará no álcool hidratado, usado como combustível veicular. Cumpre ressaltar que o álcool anidro, em contrapartida, é aquele misturado à gasolina.

2 Embora a matéria-prima seja originalmente distinta. Estados Unidos e China, por exemplo, derivam o etanol do milho. 
Segundo comunicado do Ipea (2010), o etanol ganhou espaço na política governamental brasileira na década de 1970 com a instituição do Proálcool, que tinha entre seus objetivos aliviar os problemas de balanço de pagamentos derivados da importação de petróleo. Com o passar dos anos, a produção nacional de petróleo aumentou e, em 1986, o governo retirou subsídios ao produto. Nessa linha cronológica, um ponto de inflexão importante se dá em 2004, com o boom na venda de carros flex-fuel, que fez o consumo do álcool combustível crescer. Pode-se confirmar essa informação através do Gráfico 1 e da Tabela 1.

Gráfico 1- Brasil: Licenciamento de automóveis por tipo de combustível

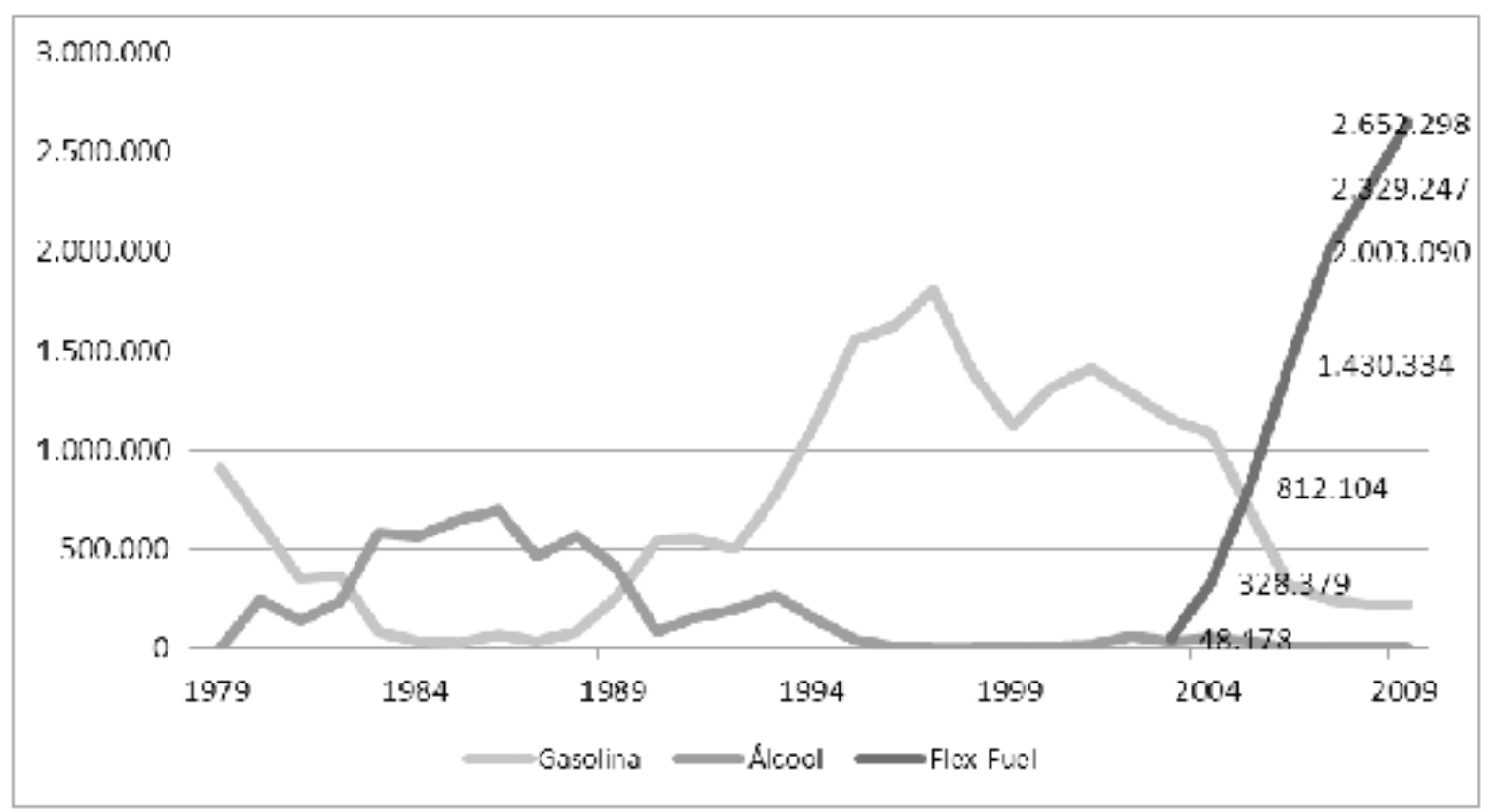

Fonte: Elaboração própria com base em dados da união da indústria de cana-de-açúcar (ÚNICA).

Nota: Similar disponível em Oñate et al. (2010).

Em 2003, quando o motor do tipo flex-fuel foi lançado, foram licenciados apenas 48.178 veículos desse tipo no Brasil. Após um curto período de consolidação, em 2004 esse número subiu para incríveis 328.379 veículos. A dinâmica foi crescente nos anos subseqüentes até superar 2,5 milhões de carros licenciados em 2009. A Tabela 1 mostra como o consumo do álcool hidratado cresceu justamente no período pós 2004:

Tabela 1- Consumo de Álcool combustível no Brasil (em milhões de litros)

\begin{tabular}{|c|c|c|}
\hline Ano & Anidro & Hidratado \\
\hline 2000 & 5.705 & 5.443 \\
\hline 2001 & 6.008 & 4.257 \\
\hline
\end{tabular}




$\begin{array}{lll}2002 & 7.250 & 4.343 \\ 2003 & 7.257 & 3.762 \\ 2004 & 7.451 & 4.835 \\ 2005 & 7.638 & 5.656 \\ 2006 & 5.200 & 7.095 \\ 2007 & 6.226 & 10.366 \\ 2008 & 6.294 & 13.290\end{array}$

Fonte: Elaboração própria com base em dados do Ministério da Agricultura, Pecuária e Abastecimento (MAPA).

Nota: Anuário estatístico da Agroenergia 2009.

É provável, portanto, que a série de preços do etanol combustível seja continuamente afetada por esse crescente consumo puxado pelo boom dos carros flex-fuel. De qualquer forma, é importante ressaltar que tal volatilidade pode ser afetada por diversos outros fatores. Desde o comportamento de produtos substitutos, como os derivados do petróleo, até o comportamento dos preços do açúcar, resultado da mesma matéria-prima. Shikida et al. (2007) por exemplo, analisam econometricamente as ofertas de álcool e açúcar do Paraná, evidenciando que a quantidade ofertada de açúcar varia inversamente ao preço do álcool. Já Serra e Zilberman (2009), através da análise de cointegração e do GARCH multivariado, demonstram que o preço do petróleo bruto impacta não apenas o nível de preços do etanol, mas também sua volatilidade.

A volatilidade dos preços do etanol tem se tornado uma preocupação crescente. Desde 2009 o governo acena com a possibilidade de formação de estoques reguladores desse produto, tendo por objetivo reduzir as oscilações de preço. Segundo o Ipea (2010), pelo menos três fatores que possibilitam maior equilíbrio nos preços do etanol brasileiro recentemente:

[...] O primeiro é a retomada dos investimentos para a formação de estoques reguladores, que tendem a equilibrar a sazonalidade. $O$ segundo foi a recente aprovação, pela Agência Nacional do Petróleo, Gás Natural e Biocombustíveis (ANP), da entrada de novos agentes e a criação de empresas de comercialização do etanol, até então proibidas pelas regras vigentes no mercado de combustíveis. O terceiro é o fato de o carro flex-fuel significar para o consumidor a possibilidade de escolha entre dois bens substitutos no momento de abastecer, gasolina e etanol, o que é um atrativo de mercado importante na consolidação destes motores. (INSTITUTO DE PESQUISA ECONÔMICA APLICADA, 2010, p. 11-12). 
A mencionada resolução da ANP que instituiu os agentes de comercialização data do final de 2009. A medida cria regras para a comercialização do etanol com o objetivo de ampliar a concorrência e estimular a formação de estoques. Nesse caso, o resultado seria uma queda na volatilidade. Sobre esse aspecto, Oñate et al. (2010) utilizam a metodologia proposta por Harwood et al. (1999) para verificar a volatilidade dos preços do etanol hidratado no estado de São Paulo no período de 2004 a 2009. Os resultados indicam grande variação nos preços e grande variabilidade na volatilidade, que foi de 52,3\% em 2004 e de apenas $22,04 \%$ em 2008. Como o cálculo de 2009 denotou volatilidade de $34,58 \%$, argumenta-se que perdas na produção da cana-de-açúcar e a crise financeira de 2008 incrementaram a volatilidade dos preços do etanol. Os autores acrescentam que:

Com base nesses cálculos, mostra-se que há uma grande incerteza com relação aos preços do mercado de etanol hidratado no estado de São Paulo. Dessa forma, trabalhos que possam auxiliar na compreensão dos instrumentos de administração de risco de preços, para tomadores de decisão nas empresas que operam com a comercialização desse produto, podem ser muito úteis. (OÑATE et al., 2010, p. 6).

De fato, a aplicação de modelos de heterocedasticidade condicional da família $\mathrm{ARCH}$ pode evidenciar a dinâmica da volatilidade e auxiliar os mecanismos de administração de risco citados por Oñate et al. (2010). Do histórico comentado até aqui, percebe-se que essa dinâmica pode ser diferenciada de acordo com o período em análise. Até 2004, quando a série não é fortemente influenciada pelo boom dos carros flex-fuel, é provável que a volatilidade apresente maior persistência, conforme sugere o Ipea (2010). No entanto, a partir de 2009 a dinâmica é aparentemente dúbia. Embora esse mesmo trabalho do Ipea advogue maior equilíbrio dos preços a partir dos três mecanismos citados anteriormente, Oñate et al. (2010) apresenta as perdas de produção da cana-de-açúcar e a crise de 2008 como impulsionadores da volatilidade.

Dito isso, o objetivo desse artigo é aplicar os modelos de volatilidade condicional à série de preços semanais do etanol hidratado no estado de São Paulo, maior produtor brasileiro, para o período de 2000 a 2011. Para isso serão considerados três períodos distintos, além da série em sua totalidade (considerado como período (a)): (b) 2000/2004 visando captar a dinâmica da volatilidade antes do impacto provocado pelos carros flex-fuel; (c) 2004/2009: visando captar a dinâmica da volatilidade depois do impacto provocado pelos carros flex-fuel; (d) 2009/2011: visando captar a dinâmica da volatilidade depois da crise e após a atuação dos meca-nismos equilibradores evidenciados pelo Ipea (2010). Pelos fatos discutidos até aqui, espera-se que a volatilidade associada ao período (ii) seja mais persistente em relação ao período (iii). Quanto ao período (iv), pode-se descobrir 
se os fatores impulsionadores da volatilidade apresentados por Oñate et al. (2010) superaram os mecanismos de equilíbrio defendidos pelo Ipea (2010).

\section{Metodologia}

Serão utilizados dados do Centro de Estudos Avançados em Economia Aplicada (CEPEA/ESALQ) para a série de preços do etanol hidratado recebidos pelo produtor no estado de São Paulo. Os dados seguem a medida $R$ \$litro, não consideram fretes nem impostos e a periodicidade é semanal. A série inicia em 07/07/2000 e segue até 27/05/2011, totalizando 569 observações. Embora a separação da série a partir de eventos de interesse tenha sido discutida na seção anterior, a divisão em períodos específicos será determinada através da análise preliminar.

Sabe-se que, em geral, a série em retornos apresenta estatísticas desejáveis como a estacionaridade e ergodicidade ${ }^{3}$. Sendo assim, inicialmente serão utilizados dados dos retornos. De todo modo, as propriedades das séries de preços e retornos serão avaliadas posteriormente. Em suma, a transformação em retornos será realizada através da primeira diferença entre os logs dos preços em períodos distintos. Ou seja: $X_{t}=\ln \left(P_{\mathrm{p}}\right)-\ln \left(\mathrm{P}_{\mathrm{t}-\mathrm{I}}\right)$.

\subsection{Modelos Básicos de Volatilidade: ARCHe GARCH}

Se o objetivo é modelar volatilidade de uma série financeira, é preciso considerar os seguintes fatos estilizados na literatura (PEÑA et al., 2001 apud MORETTIN; TOLOI, 2006): (a) a volatilidade aparece em grupos; (b) a volatilidade é estacionária; (c) a volatilidade reage diferente a choques positivos e negativos; (d) exibem não linearidades na variância. Sob esse aspecto, Enders (2004) chama atenção para um ponto importante: em modelos econométricos básicos, assumese que a variância do termo de erro é constante. No entanto, geralmente as séries financeiras possuem períodos de alta volatilidade (agrupamentos de volatilidade) seguidos por período de relativa tranquilidade.

Os modelos ARCH (p) atuam especificamente no ponto (iv) ao pressupor que a variância condicional se altera no tempo. Assume-se que a volatilidade de uma série temporal é uma variável aleatória condicionada pela variabilidade verificada nos momentos passados. De acordo com Morettin e Toloi (2006), em finanças a ideia básica é que o retorno é não correlacionado serialmente, mas a volatilidade (variância condicional) depende de retornos passados por meio de uma função quadrática. Assim, um modelo ARCH (p) é definido por:

3 Detalhes podem ser consultados em Campbell et al. (1997). 


$$
\begin{aligned}
& X_{t}=\sqrt{h_{t} \varepsilon_{t}} \\
& h_{t}=\alpha_{0}+\alpha_{1} X_{t-1}^{2}+\ldots+\alpha_{r} X_{t-r}^{2}
\end{aligned}
$$

Onde se supõe $\alpha_{0}>0, \alpha_{i} \geq 0, \varepsilon \sim N(0,1)$ ou $\varepsilon_{t} \sim t_{v}$. Note que, se apenas $\alpha_{0} \neq 0$, a variância estimada é simplesmente essa constante. Caso contrário, ela evolui como um processo autoregressivo.

Segundo Tsay (2005), o procedimento para a construção de um modelo de volatilidade consiste em quatro passos:

a) especificar uma equação para a média através do teste de dependência serial nos dados e, se necessário, construir um modelo econométrico (ARMA) para remover essa dependência;

b) usar os resíduos do modelo empregado para testar efeitos $\mathrm{ARCH}$;

c) especificar um modelo de volatilidade caso os efeitos $\mathrm{ARCH}$ sejam estatisticamente significativos;

d) fazer uma verificação cuidadosa do modelo e refiná-lo, caso necessário.

No caso de séries financeiras de retornos diários, é normal que a modelagem referente ao passo:

a) seja realizada através de um modelo autorregressivo simples. $\mathrm{O}$ ponto

b) pode ser efetuado a partir do teste de Box-Pierce-Ljung ou via multiplicadores de Lagrange (ML) de Engle (1982).

A principal vantagem associada ao modelo $\mathrm{ARCH}$ é que admite caudas mais pesadas que a normal. No entanto, em geral, a aplicação desse método requer a consideração de um grande número de parâmetros. Uma generalização dos modelos ARCH foi proposta por Bollerslev (1986) através dos modelos GARCH, que podem ser utilizados para descrever a volatilidade com menos parâmetros. Em suma, um modelo GARCH $(p, q)$ é definido por:

$$
\begin{aligned}
& X_{t}=\sqrt{h_{t} \varepsilon_{t}} \\
& h_{t}=\alpha_{0}+\sum_{i=1}^{r} \alpha_{i} X_{t-i}^{2}+\sum_{j=1}^{s} \beta_{j} h_{t-j}
\end{aligned}
$$

Assume-se que $\alpha_{0}>0, \alpha_{i} \geq 0, \beta_{j} \geq 0, \sum_{i=1}^{q}\left(\alpha_{i}+\beta_{j}\right)<1$.Nesse caso, $\alpha 1$ é o coeficiente de reação da volatilidade e mede $a$ extensão com que um choque no retorno em $t$ influencia a volatilidade do retorno em $t+1$. Já $\beta 1$ mede o tempo que um choque na variância condicional leva para dissipar-se. Em suma, $\alpha 1+\beta 1$ mede, de forma geral, a velocidade com que os choques na volatilidade são removidos 
para cada defasagem. Note que, mesmo com uma estrutura mais parcimoniosa, esse modelo permite maiores informações sobre a série em relação à aplicação de uma estratégia $\mathrm{ARCH}(\mathrm{p})$.

\subsection{Modelos Assimétricos: EGARCH e TARCH}

Apesar dos avanços, os modelos GARCH não estão isentos de críticas. Tanto o ARCH quanto GARCH não consideram o terceiro fato estilizado mencionado anteriormente: a volatilidade reage diferente em relação a choques positivos e choques negativos. Ou seja, as séries são caracterizadas por efeitos assimétricos. Enquanto períodos de queda (associados a choques negativos) são caracterizados por grande volatilidade, períodos de ascensão (associados a choques positivos) caracterizam volatilidade mais previsível. Sendo assim, é necessário utilizar modelos capazes de considerar essa diferenciação e indicar se há efeito alavancagem na série. Ou seja, se choques negativos tem efeito maior que os choques positivos.

Para isso, pode-se utilizar o modelo EGARCH ("Exponential GARCH") introduzido por Nelson (1991):

$$
\ln \left(\sigma_{t}^{2}\right)=w+\beta_{1} \ln \left(\sigma_{t-1}^{2}\right)+\alpha_{1}\left(\frac{\left|\varepsilon_{t-1}\right|}{\sqrt{\sigma_{t-1}^{2}}}-\sqrt{\frac{2}{\pi}}\right)+\gamma_{1}\left(\frac{\varepsilon_{t-1}}{\sqrt{\sigma_{t-1}^{2}}}\right)
$$

Onde $\alpha$ lé o coeficiente de reação e $\beta_{1}$ é o coeficiente de persistência. Agora o modelo expõe explicitamente um parâmetro, $\gamma_{1}$, para captar a assimetria. Caso $\gamma_{1}=0$, um choque positivo tem o mesmo efeito na volatilidade que um choque negativo de mesma magnitude. Se $\gamma_{1} \neq 0$ existe assimetria. Se, além disso, $\gamma_{1}<0$, há efeito alavancagem e choques negativos causam maior impacto que choques positivos. Segundo Hamilton (1994), uma das principais vantagens associadas a essa abordagem está em utilizar o log da variância condicional. Nesse caso, a variação em si será positiva, independente do sinal dos parâmetros. Nesse sentido, ao contrário do modelo GARCH, não precisam ser realizadas restrições em (3) para estimação.

Outro modelo que capta a assimetria é o modelo TARCH ("Threshold ARCH") introduzido por Zakoian (1994). Segundo Moretin e Toloi (2006), trata-se de um caso particular do modelo ARCH não linear onde a variância condicional segue a forma funcional:

$$
\sigma_{t}^{2}=\alpha_{0}+\alpha_{1} \varepsilon_{t-1}^{2}+\beta_{1} \sigma_{t-1}^{2}+\gamma_{1} d_{t-1} \varepsilon_{t-1}^{2}
$$

Nesse caso, $\alpha_{0}, \alpha_{1}$ e $\beta_{1}$ são parâmetros não negativos. A novidade está em utilizar $d_{\mathrm{t}-1}$, uma dummy que assume valor 1 caso $\varepsilon_{t-1}<0$ (más notícias) e 0 caso 
$\varepsilon_{t-1}>0$ (boas notícias). Se $\gamma_{1} \neq 0$, há assimetria e choques positivos e negativos possuem impacto diferenciado. Se $\gamma_{1}>0$, há efeito alavancagem. Note que, ao utilizar valor 1 para más notícias, o modelo capta justamente o impacto que choques negativos impõem à série, sendo possível, inclusive, quantificá-lo. Percebe-se que choque negativos $\left(\varepsilon_{t-1}^{2}<0\right)$ promovem impacto igual a $\alpha_{1}+\gamma_{1}$ na volatilidade, enquanto choques positivos $\left(\varepsilon_{t-1}^{2}>0\right)$ promovem impacto equivalente a $\alpha_{1}$. Isso deixa claro, adicionalmente, que o modelo supõe alavancagem a priori.

\subsection{Modelos Fracionários na Média e na Variância: ARFIMA-FIGARCH}

Comentou-se na seção 3.1 que o primeiro passo na construção de um modelo de volatilidade é especificar um processo ARMA $(p, q)$ para a média condicional visando modelar a correlação existente. Essa estratégia admite a série estacionária, uma vez que o parâmetro de diferenciação é zero ${ }^{4}$. Esse procedimento é comumente aplicado na série de retornos, após um teste padrão de estacionaridade onde essa característica é verificada. No entanto, essa estratégia está sujeita a sobrediferenciação, uma vez que o cálculo dos retornos equivale à primeira diferença e $d=1$ pode denotar uma diferenciação excessiva e pouco parcimoniosa.

Seguindo Morettin e Toloi (2006), $\left\{Z_{t}\right\}$ é um processo auto-regressivo fracionário integrado de médias móveis, ou seja, ARFIMA (p,d,q) com $d \epsilon(-1 / 2,1 / 2)$ se for estacionário e satisfizer

$$
\phi(B)(1-B)^{d_{m}} Z_{t}=\theta(B) a_{t}
$$

Onde $\left\{a_{t}\right\}$ é um ruído branco e $\phi(B)$ e $\Theta(B)$ são polinômios em $B$ de graus $p$ e $q$, respectivamente. Nesse caso, $d_{m}$ explica a estrutura de correlação de ordens altas da série, ou seja, a memória de longo prazo da média condicional, enquanto $\phi$ e $\Theta$ explicam a estrutura de correlação de ordens baixas. Nesse caso, a estrutura ARMA modela o comportamento de curto prazo e o parâmetro $d_{m}$ repercute a dinâmica de longo prazo. Em geral, se $d_{m}<1 / 2$ a série é estacionária. O método se deve a Granger e Joyeux (1980) e Hosking (1981), referências básicas para informações adicionais.

Situação análoga à descrita anteriormente pode ser estendida à variância condicional. Considere os modelos $\mathrm{GARCH}(\mathrm{p}, \mathrm{q})$ tradicionais onde a soma dos coeficientes, $\alpha+\beta$, é próximo de 1 , indicando grande nível de persistência. Para superar essa situação de persistência excessiva, o modelo FIGARCH (p,d,q) foi proposto por Baillie, Bollerslev e Mikkelsen (1996). Visando abordar melhor o problema, defina inicialmente o modelo IGARCH $(\mathrm{p}, \mathrm{q})$ como

$4 \quad$ Note que um processo ARMA $(\mathrm{p}, \mathrm{q})$ equivale a um ARIMA $(\mathrm{p}, \mathrm{d}, \mathrm{q})$, onde $\mathrm{d}=0$ nesse último. 


$$
\phi(B)(1-B) X_{t}^{2}=w+[1-\beta(B)]\left(X_{t}^{2}-\sigma_{t}^{2}\right)
$$

com $\phi(B)=[1-\alpha(B)-\beta(B)](1-B)^{-1}$. Substituindo o operador de primeira diferença $(1-B)$ pelo operador de diferença fracionária $(1-B)^{d_{\nu}}$, tem-se o modelo FI$\mathrm{GARCH}(\mathrm{p}, \mathrm{d}, \mathrm{q})$ onde a variância condicional toma a forma

$$
\sigma_{t}^{2}=w[1-\beta(B)]^{-1}+\left\{1-[1-\beta(B)]^{-1} \phi(B)(1-B)^{d_{v}}\right\} X_{t}^{2}
$$

Com $0 \leq d_{v} \leq 1$. Se, nos modelos GARCH (p,q), $\alpha+\beta$ é próximo de 1, o decaimento de choques na variância condicional é muito lento, o que equivale a uma alta persistência. No entanto, o decaimento exponencial proposto pelo GARCH $(p, q)$ pode ser muito rápido no processo de captura da dependência. Nesse caso, considera-se um decaimento hiperbólico como o proposto pelo modelo FIGARCH $(p, d, q)$. Nesse contexto, os parâmetros do modelo GARCH $(p, q)$ padrão representam a persistência de curto prazo, enquanto o parâmetro de diferenciação fracionária $d_{v}$ representa a longa memória do processo, ou seja, a persistência de longo prazo da variância condicional. Embora a proposta de aplicação dos parâmetros de diferenciação no modelo ARFIMA (p,d,q) e no modelo FIGARCH $(p, d, q)$ derivem de preocupação idêntica, a leitura dos resultados é diferenciada no que se refere à magnitude do parâmetro $d$. No ARFIMA $(p, d, q)$, quanto maior o parâmetro, maior a memória do processo. No FIGARCH (p,d,q), ao contrário, maiores parâmetros de diferenciação identificam menores níveis de persistência.

\section{Resultados}

O primeiro passo consiste em uma análise preliminar da série de preços e retornos. A série de preços pode ser visualizada no Gráfico 2. O eixo das abscissas corresponde ao período, onde 01 denota 2001, 02 representa 2002 e assim por diante. O eixo das coordenadas constitui o preço do etanol em $\mathrm{R} \$ /$ litro. Em termos estatísticos, suspeita-se da não estacionaridade da série, uma vez que ela não se desenvolve ao redor de uma média e sim apresenta tendência ascendente. Essa suspeita é corroborada ${ }^{5}$ a partir dos testes ADF (Augmented Dickey-Fuller) e PP (Phillips-Perron) de raiz unitária disponíveis no apêndice A2. Cumpre ressaltar que a média de preço do etanol hidratado no estado de São Paulo durante o período analisado é de $\mathrm{R} \$ 0,744$ por litro.

A primeira informação gráfica é a grande variabilidade na série de preços. Comportamento natural em se tratando de uma commodity internacional, afetada por diversos fatores, conforme comentado anteriormente. A série inicia com

5 A 1\%, não se rejeita a hipótese nula de raiz unitária em nenhum dos testes utilizados. 
uma queda que é interrompida na segunda metade de 2002. Inicia-se então uma dinâmica ascendente que dura até maio de 2003. Segundo o relatório ${ }^{6}$ Agromensal da CEPEA/ESALQ sobre açúcar e álcool referente a janeiro de 2003, o aumento da demanda interna em 2002 é responsável pela elevação dos preços do etanol nos meses anteriores ao relatório. Durante esse período, cresceu a venda de carros a álcool e houve elevação nos preços da gasolina. Esse tipo de análise pode ser estendido a todo o período na série, indicando de que modo choques específicos influenciaram conjunturalmente a série de preços.

Gráfico 2- Série de preços do etanol no Estado de São Paulo, 2000/201 1

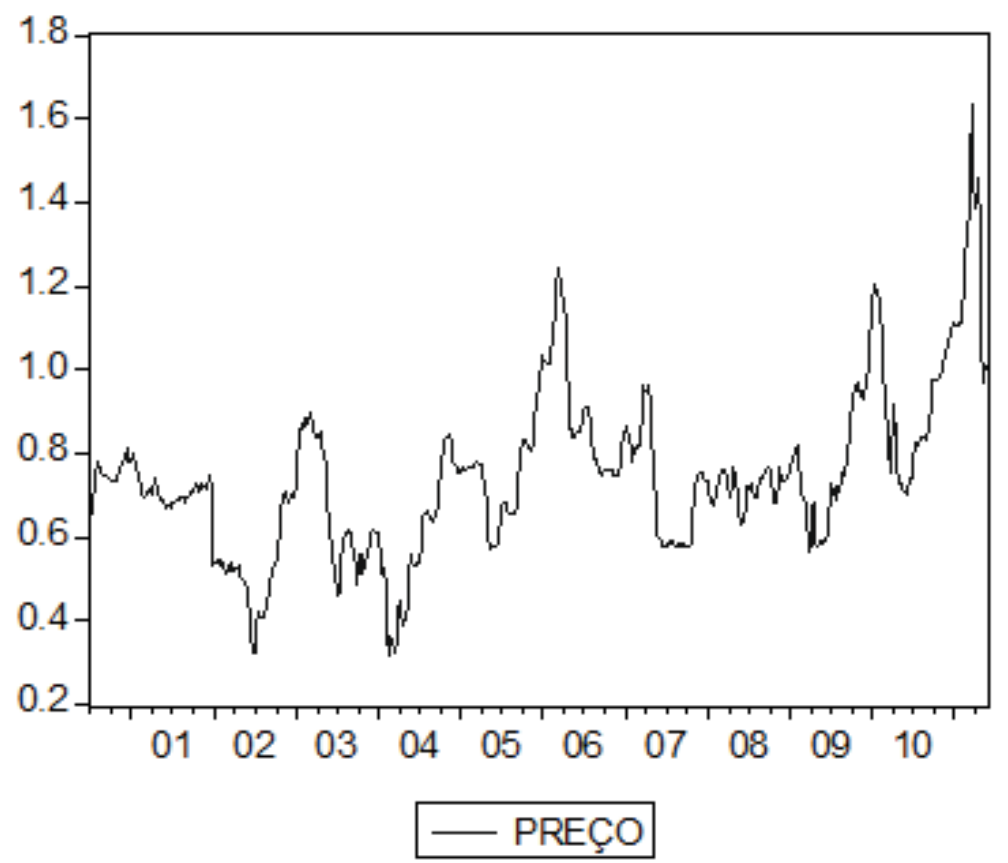

Fonte: Elaboração própria.

Informações adicionais podem ser captadas analisando o período como um todo e verificando a dinâmica que a série assume independente de choques específicos. Apesar dos choques positivos, é possível notar uma tendência descendente na série de preços durante o período inicial até a terceira semana de fevereiro em 2004 , quando o preço do etanol alcançou o piso de $\mathrm{R} \$ 0,3186$ por litro, menor valor da série. A partir de então, a série alcançou trajetória ascendente, mesmo que levemente interrompida durante o ano de 2008. Aqui provavelmente se estabelece o impacto promovido pelo boom dos carros flex-fuel iniciado em 2004. Notase que as oscilações permanecem, mas a um patamar mais elevado de preços. Prova disso é que o menor valor assumido a partir de então é de $\mathrm{R} \$ 0,5654$ por litro, na primeira semana de abril em 2009. A média de preços até a terceira se-

6 Disponível em: <http://www.cepea.esalq.usp.br/agromensal/2003/01_janeiro/acucaralcool.htm> . 
mana de fevereiro de 2004 é de $\mathrm{R} \$ 0,6454$ por litro. A partir daí até o final da série, a média de preços é de $\mathrm{R} \$ 0,7943$ por litro. Esses dados demonstram que há realmente uma dinâmica diferenciada durante o período inicial, anterior ao impacto provocado pelos carros flex-fuel, e o período subsequente.

No trecho final da série a dinâmica assume o seguinte movimento: os preços começam a declinar a partir da primeira semana de fevereiro em 2009. Nesse momento, o preço que era $R \$ 0,8208$ por litro cai até atingir $R \$ 0,5654$ por litro na primeira semana de abril em 2009. Segundo Jank (2010), a crise econômica promoveu falta de liquidez no mercado de crédito durante o primeiro semestre de 2009, "[...] forçando boa parte das empresas a desovar grandes volumes de etanol a preços fortemente deprimidos, abaixo dos custos de produção, para poderem se capitalizar". A partir desse período o movimento é ascendente até atingir o pico da série na quarta semana de março em 2011 , quando o preço chegou a $R \$ 1,63$ por litro. Em suma, a partir do piso atingido na primeira semana de abril em 2009, a série alcançou uma dinâmica ascendente até atingir o pico mencionado. Nesse momento, o preço volta a cair e atinge $R \$ 0,9680$ por litro na segunda semana de maio em 2011.

Gráfico 3 - Série de retornos do etanol no estado de São Paulo, 2000/2011

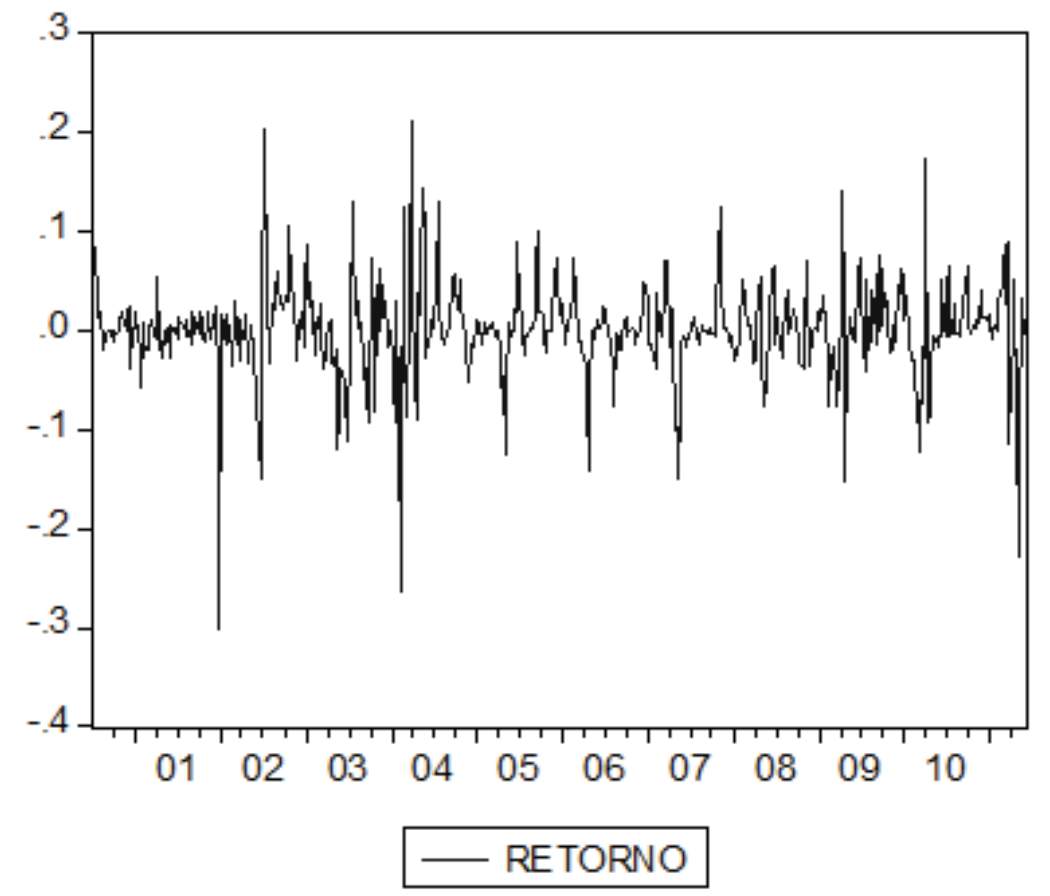

Fonte: Elaboração própria.

Outros detalhes podem ser captados analisando o Gráfico 2, que representa a série de retornos. Em termos estatísticos, confirma-se o fato estilizado na literatura: os retornos são estacionários (ver apêndice A2). Outro fato estilizado é perceptível 
graficamente: agrupamentos de volatilidade. Existem agrupamentos perceptíveis no começo da série até o início de 2004. De 2004 a 2009, não existem agrupamentos perceptíveis. No entanto, esses parecem retornar, em menor medida, no período final. Essa característica é um indicio da presença de efeitos $\mathrm{ARCH}$.

Em suma, o gráfico dos retornos parece corroborar uma divisão da série em três períodos distintos. Tomando por base todas as informações aqui discutidas, utilizam-se com os seguintes períodos: (i) 2000/2011: ou seja, a série em sua totalidade, com o objetivo de verificar a dinâmica geral da volatilidade dos preços do etanol durante o período em análise; (ii) 07/07/2000 a 20/02/2004: visando verificar a dinâmica da volatilidade no período de declínio do preço, antes do boom dos carros flex-fuel, quando o regime de preços se dava em um nível médio inferior; (iii) 27/02/2004 a 06/02/2009: após o boom de carros flex-fuel, evento que teoricamente ajudou a promover a estabilidade da série e (iv) 13/02/2009 a 27/05/2011: quando o governo passou a se preocupar mais fortemente com a volatilidade dos preços do etanol e quando, segundo o Ipea (2010), passaram a atuar outros mecanismos de manutenção do equilíbrio de preços.

A análise inicial considerará a série de retornos. Apesar do teste de JarqueBera rejeitar a hipótese de normalidade nas distribuições de preços e retornos (ver apêndice A1), os testes de raiz unitária indicaram estacionaridade nesta última, ao contrário da primeira, corroborando os fatos estilizados na literatura, segundo o qual os retornos apresentam características estatísticas mais desejáveis.

\subsection{Modelos Básicos: GARCH, EGARCHE TARCH}

Utilizou-se o procedimento padrão para modelar a média condicional da série. Ou seja, as funções de autocorrelação e autocorrelação parcial foram utilizadas como guia na definição da modelagem $\operatorname{ARMA}(p, q)$ para média condicional, visando remover a autocorrelação serial entre as observações. A informação decisiva, no entanto, se deu a partir das estatísticas de informação AIC e SBC, considerando ainda o critério da parcimônia.

A primeira análise se deu na série em sua totalidade. O estudo do correlograma da série de retornos demonstrou que os retornos são autocorrelacionados. Essa característica foi modelada a partir de um modelo ARMA $(2,0)$ na média condicional. Após essa modelagem, os testes de autocorrelação e a análise do correlograma indicaram a ausência de autocorrelação nos resíduos. No entanto, o ARCH LM Test retornou p-valor igual a zero, indicando a presença de efeitos $\mathrm{ARCH}$, conforme os agrupamentos de volatilidade pareciam indicar. Sendo assim, partiu-se para os modelos da família ARCH visando modelar a variância condicional. Os resultados estão sumarizados na Tabela 2.

7 As estimações foram realizadas no Software Eviews. 
Os resíduos foram testados novamente e em todos os casos detectou-se a ausência de efeitos ARCH. Ou seja, os modelos utilizados modelaram a variância condicional. Os resultados para os demais períodos podem ser consultados no apêndice B1, B2 e B3, respectivamente. Captou-se efeito alavancagem na série inteira e no período (ii), informando que choques negativos promovem maior impacto que choques positivos nesses períodos. No período (iii) captou-se assimetria sem efeito alavancagem e no período (iv) não parece haver efeitos assimétricos. O que chama grande atenção é um alto componente de reação a choques e o somatório dos parâmetros maior que 1, indicando uma provável presença de longa memória na variância condicional para todos os períodos. Esses resultados corroboram a utilização de modelos da família FIGARCH (p,d,q).

Tabela 2 - GARCH, EGARCH e TARCH para a série de retornos, 2000/2011

\begin{tabular}{|c|c|c|c|}
\hline Variância & GARCH $(\mathbf{1}, \mathbf{1})$ & $\operatorname{EGARCH}(1,1)$ & $\operatorname{TARCH}(1,1)$ \\
\hline$\alpha_{0}$ & $\begin{array}{l}0.0002^{* * *} \\
(0.0000)\end{array}$ & $\begin{array}{c}-2.9220 * * * \\
(0.0000)\end{array}$ & $\begin{array}{l}0.0002^{* * *} \\
(0.0000)\end{array}$ \\
\hline$\varepsilon_{t-1}^{2}$ & $\begin{array}{l}1.2914 * * * \\
(0.0000)\end{array}$ & & $\begin{array}{l}1.1235^{* * *} \\
(0.0000)\end{array}$ \\
\hline$\sigma_{t-1}^{2}$ & $\begin{array}{c}0.1565 * * * \\
(0.0000)\end{array}$ & & $\begin{array}{c}0.1671^{* * *} \\
(0.0000)\end{array}$ \\
\hline$d_{t-1} \varepsilon_{t-1}^{2}$ & & & $\begin{array}{l}0.2653^{*} \\
(0.0887)\end{array}$ \\
\hline$\left|\varepsilon_{t-1}^{2}\right| /\left|\sigma_{t-1}\right|$ & & $\begin{array}{c}1.1422^{* * *} \\
(0.0000)\end{array}$ & \\
\hline$\varepsilon_{t-1}^{2} / \sigma_{t-1}$ & & $\begin{array}{c}-0.1601^{* * *} \\
(0.0000)\end{array}$ & \\
\hline $\ln \left(\sigma_{t-1}^{2}\right)$ & & $\begin{array}{c}0.6842 * * * \\
(0.0000)\end{array}$ & \\
\hline ARCH LM Test & 0.4661 & 0.3745 & 0.4487 \\
\hline $\mathrm{AIC}$ & -3.9629 & -3.9586 & -3.9610 \\
\hline SBC & -3.9247 & -3.9127 & -3.9151 \\
\hline
\end{tabular}

Fonte: Elaboração própria.

Nota: No caso do ARCH LM Test, os valores correspondem ao p-valor. Para os demais, o p-valor aparece entre parênteses.

\subsection{ARFIMA-FIGARCH 8}

Na seção anterior utilizou-se a série de retornos do etanol e empregou-se uma estrutura do tipo ARMA (p, q) para modelar a média condicional. Essa estra-

8 As estimações foram realizadas no Software Matrixer. 
tégia admite raiz unitária na série de preços e pode promover sobrediferenciação visando obter dados estacionários via retornos. Uma estratégia mais parcimoniosa é utilizar modelos do tipo ARFIMA (p, d, q) na série de preços de forma a obter uma diferenciação na medida adequada, promovendo uma modelagem mais limpa na média condicional. Percebeu-se ainda que, na aplicação dos modelos padrões da família $\mathrm{ARCH}$, os somatórios dos parâmetros indica a presença persistência de longo prazo na variância condicional, de forma que a aplicação de modelos fracionários na variância torna-se adequada. Sendo assim, a Tabela 3 apresenta resultados para a aplicação do modelo ARFIMA-FIGARCH na série de preços do etanol nos quatro períodos em análise.

A primeira informação crucial está na significância dos parâmetros de diferenciação para a média condicional. Fica claro que utilizar os retornos implica sobrediferenciar a série, especialmente para os dois períodos finais, quando $d_{m}<0,5$. Os p-valores do ARCH LM Test indicam que a heterocedasticidade condicional foi modelada em todos os períodos.

Outro ponto, esse fundamental para o teste da proposta empírica estabelecida, está na significância do parâmetro de diferenciação fracionária para a variância condicional. De fato, conforme suspeita anterior, existe persistência na variância condicional, uma vez que os parâmetros foram positivos e significativos. Convém relembrar que a análise do parâmetro $d$ no FIGARCH $(p, d, q)$ é inversa àquela realizada no ARFIMA (p,d,q). Nesse último caso, quando maior o parâmetro, maior a persistência. No primeiro, quanto menor o parâmetro, maior a persistência. Sendo assim, os resultados demonstram que a persistência na variância condicional é consideravelmente forte quando se analisa a série em sua totalidade. Interessante ver que, para o período (ii), a memória de longo prazo da volatilidade é bastante elevada, principalmente em comparação ao período subseqüente. $\mathrm{O}$ maior parâmetro de diferenciação para o período (iii) indica que, nesse momento, a memória de longo prazo da volatilidade é menor e um choque na volatilidade demora menos pra se dissipar. Esse resultado corrobora a teoria de que a entrada dos carros flex-fuel a partir de 2004 funcionou como mecanismo de equilíbrio dos preços.

Tabela 3 - ARFIMA-FIGARCH para a série de preços do etanol

\begin{tabular}{ccccc}
\hline Parâmetro & (i)2000/2011 & (ii)2000/2004 & (iii)2004/2009 & (iv)2009/201 1 \\
\hline \multirow{2}{*}{ Constante } & $0.6236^{* * *}$ & $0.3876^{* * *}$ & $0.7070^{* * *}$ & $1.0326^{* * *}$ \\
& $(0.0000)$ & $(0.0000)$ & $(0.0000)$ & $(0.0000)$ \\
\multirow{2}{*}{$\mathrm{AR}(1)$} & $0.5812^{* * *}$ & $-0.0974^{* * *}$ & $1.1162^{* * *}$ & $0.9081^{* * *}$ \\
& $(0.0000)$ & $(0.0000)$ & $(0.0000)$ & $(0.0000)$ \\
\hline
\end{tabular}




\begin{tabular}{|c|c|c|c|c|}
\hline Parâmetro & (i) $2000 / 2011$ & (ii)2000/2004 & (iii)2004/2009 & (iv)2009/2011 \\
\hline $\mathrm{AR}(2)$ & & $\begin{array}{c}0.5332^{* * *} \\
(0.0000)\end{array}$ & $\begin{array}{c}-0.1123 * * * \\
(0.0000)\end{array}$ & \\
\hline $\mathrm{MA}(1)$ & & $\begin{array}{c}0.6136 * * * \\
(0.0000)\end{array}$ & $\begin{array}{c}0.1398^{* * * *} \\
(0.0000)\end{array}$ & $\begin{array}{c}0.1708^{* * *} \\
(0.0000)\end{array}$ \\
\hline $\mathrm{MA}(2)$ & & & $\begin{array}{c}0.0362^{* * *} \\
(0.0000)\end{array}$ & \\
\hline$d_{m}$ & $\begin{array}{c}0.8933^{* * * *} \\
(0.0000)\end{array}$ & $\begin{array}{c}0.9387 * * * \\
(0.0000)\end{array}$ & $\begin{array}{c}0.1098 * * * \\
(0.0000)\end{array}$ & $\begin{array}{c}0.3833 * * * \\
(0.0000)\end{array}$ \\
\hline ARCH Const. & $\begin{array}{c}0.0090 * * * \\
(0.0000)\end{array}$ & $\begin{array}{c}0.0109 * * * \\
(0.0000)\end{array}$ & $\begin{array}{c}0.0059 * * * \\
(0.0000)\end{array}$ & $\begin{array}{c}0.0063 * * * * \\
(0.0000)\end{array}$ \\
\hline $\mathrm{ARCH}(1)$ & $\begin{array}{c}0.9622 * * * \\
(0.0000)\end{array}$ & $\begin{array}{c}0.9682^{* * *} \\
(0.0000)\end{array}$ & $\begin{array}{c}0.8308^{* * * *} \\
(0.0000)\end{array}$ & $\begin{array}{c}0.8858^{* * *} \\
(0.0000)\end{array}$ \\
\hline GARCH(1) & $\begin{array}{c}0.1956 * * * \\
(0.0000)\end{array}$ & $\begin{array}{c}0.2074 * * * \\
(0.0000)\end{array}$ & $\begin{array}{c}0.2414 * * * \\
(0.0000)\end{array}$ & $\begin{array}{c}0.2624^{* * *} \\
(0.0000)\end{array}$ \\
\hline$d_{v}$ & $\begin{array}{c}0.1011^{* * *} \\
(0.0000)\end{array}$ & $\begin{array}{c}0.1369 * * * \\
(0.0000)\end{array}$ & $\begin{array}{c}0.3931 * * * \\
(0.0000)\end{array}$ & $\begin{array}{c}0.2210 * * * \\
(0.0000)\end{array}$ \\
\hline ARCH LM Test & 0.2815 & 0.9769 & 0.3983 & 0.2057 \\
\hline AIC & -4.6647 & -4.8530 & -5.0686 & -3.9733 \\
\hline SBC & -4.6112 & -4.6981 & -4.9305 & -3.7864 \\
\hline
\end{tabular}

Fonte: Elaboração própria.

Nota: No caso do ARCH LM Test, os valores correspondem ao p-valor. Para os demais, o p-valor aparece entre parênteses.

Para o período (iv) o parâmetro de diferenciação é maior que no período (ii), mas menor que no período (iii). Ou seja, no momento final pós 2009 os choques são mais persistentes em comparação ao período imediatamente anterior à crise e aos mecanismos de equilíbrio evidenciados pelo Ipea (2010). Não é exatamente uma surpresa: a regulamentação dos agentes de comercialização e os estímulos à formação de estoques remetem ao final de 2009 e suas influências sobre a persistência da volatilidade certamente não estão plenamente estabelecidas. Ao contrário, por exemplo, do impacto advindo da crise econômica em 2008 evidenciado por Jank (2010).

Em suma, os resultados apresentam duas informações importantes: (i) a volatilidade dos preços do etanol é bastante persistente. Captou-se a presença de longa memória na variância condicional dos preços. O parâmetro de diferenciação significativo igual a 0.10 indica uma grande memória de longo prazo, de forma que um choque na volatilidade impacta a série por um grande período de tempo. Esse resultado corrobora a preocupação do governo não apenas com a volatili- 
dade em si, mas com a persistência que assume, indicando que os mecanismos de equilíbrio de preços, como os estoques reguladores, podem ser necessários para que choques se dissipem com mais facilidade e o preços possam permanecer em equilíbrio na maior parte do tempo; (ii) o Ipea (2010) parece correto ao colocar a entrada dos carros flex-fuel como mecanismo equilibrador. A persistência da volatilidade é menor após a entrada desse mecanismo no mercado. Mesmo que a volatilidade tenha se tornado mais persistente no período final pós 2009, provavelmente em virtude dos impactos da crise econômica evidenciados por Jank (2010), ela é consideravelmente inferior à persistência de longo prazo praticada no período inicial, quando os carros flex-fuel não estavam disponíveis no mercado e o consumo do etanol se dava em um nível médio inferior.

\section{Considerações Finais}

O principal objetivo desse estudo foi estudar a dinâmica da volatilidade dos preços do etanol no estado de São Paulo. Devido à importância do produto, a grande variação em seus níveis de preço ganhou espaço na discussão sobre biocombustíveis no Brasil e fundamentou o debate sobre a necessidade de políticas públicas capazes de promover a manutenção do equilíbrio. Além da série em sua totalidade, representando o período 2000/2011, estudou-se três sub períodos distintos com o objetivo de captar o impacto de eventos específicos.

Em suma, a metodologia empregada detectou persistência de longo prazo na volatilidade dos preços do etanol. Os resultados dão suporte às preocupações do governo com a volatilidade dos preços e com a persistência que pode assumir após os diversos choques a que estão submetidos. Nesse sentido, sustenta as discussões sobre a necessidade da formação de estoques reguladores do produto e das demais políticas econômicas que objetivam promover estabilidade. Adicionalmente, corrobora a versão do Ipea (2010) segundo a qual a entrada dos carros flex-fuel no mercado nacional colaborou para a manutenção do equilíbrio e diminuição da persistência na volatilidade dos preços do produto. Mesmo que no período recente pós-crise essa persistência tenha se tornado mais agressiva, o patamar praticado é inferior ao estabelecido no início da série.

Para auxiliar a elaboração de políticas públicas, os resultados demonstram a efetividade do mecanismo de mercado representado pelos carros flex-fuel, mas também deixam claro que os efeitos estabilizadores positivos por eles empregados podem ser mitigados por distintos mecanismos desequilibradores, o que reforça a necessidade de se estudar medidas de manutenção do equilíbrio de preços. $\mathrm{O}$ resultado para o último sub período analisado enfatiza a dificuldade de se atingir o objetivo, uma vez que um evento intrinsecamente não relacionado ao etanol (a 
crise de 2008) conseguiu impactá-lo em meio a medidas de estabilização empregadas, como a instituição dos agentes de comercialização.

Em suma, com os resultados apresentados nesse estudo e as evidências contidas em trabalhos como Shikida et al. (2007) e Serra e Zilberman (2009), o governo tem evidências suficientes para justificar e direcionar política estabilizadoras adequadas, seja via formação de estoques reguladores do produto, seja considerando o impacto de mercados específicos (como o açúcar e o petróleo) no comportamento dos preços do etanol.

\section{Referências}

ARÊDES, A. F.; PEREIRA, M. W. Potencialidade da Utilização de Modelos de Séries Temporais na Previsão do Preço do Trigo no Estado do Paraná. Revista de Economia Agrícola, São Paulo, n. 55, p.63-76, abr./jun. 2008.

BAILLIE, R.T.; BOLLERSLEV, T.; MIKKELSEN, H.O. Fractionally Integrated Generalized Autoregressive Conditional Heterocedasticity. Journal of Econometrics, Chicago, n. 74, n. 1, p. 3-30, Sep. 1996.

BOLLERSLEV, T. Generalized Autoregressive Conditional Heteroskedasticity. Journal of Econometrics, Chicago, n. 31, n. 3, p. 307-327, 1986.

BRASIL. Ministério da Agricultura, Pecuária e Abastecimento. Anuário Estatístico da Agroenergia. Brasília, 2009.

CAMPBELL, J.Y.; LO, A.W.; MACKINLAY, A. C. The Econometrics of Financial Markets. Princeton: Princeton University Press, 1997.

CAMPOS, K. C.; PIACENTI, C. A. Agroenergia: A Questão da Volatilidade de Preços e o Efeito Alavancagem dos Produtos Agrícolas. In: CONGRESSO DA SOCIEDADE BRASILEIRA DE ECONOMIA, ADMINISTRAÇÃO E SOCIOLOGIA RURAL, 45., 2007, Londrina. Anais... Londrina: SOBER, 2007.

CUNHA, C. A.; WANDER, A. E.; ARAÚJO, K. D. Volatilidade dos Preços Recebidos pela Cana-de-Açúcar no Estado de São Paulo, 1995-2007. In: CONGRESSO DA SOCIEDADE BRASILEIRA DE ECONOMIA, ADMINISTRAÇÃO E SOCIOLOGIA RURAL, 47., 2009, Porto Alegre. Anais... Porto Alegre: SOBER, 2009.

ENDERS, W. Applied Econometric Time Series. New Jersey: Wiley, 2004.

ENGLE, R. F. Autoregressive Conditional Heterocedasticity With Estimates of de Variance of UK Inflation. Econometrica, Chicago, n. 50, p. 987-1008, 1982.

GRANGER, C.W.; JOYEUX, R. A Introduction to Long Memory Time Series Models and Fractional Differencing. Journal of Time Series Analysis, Clevedon, GB, n. 1, p. 5-39, Jan. 1980.

HAMILTON, J. Time Series Analysis. Princeton: Princeton University Press, 1994.

HARWOOD, J. et al. Managing Risk in Farming: Concepts, Research and Analysis. Agricultural Economics Report, Washington, DC, n. 774, Mar. 1999.

HOSKING, J. R. Fractional Differencing. Biometrika, London, GB, v. 68, n. 1, p. 165-176, May 1981. 
INSTITUTO DE PESQUISA ECONÔMICA APLICADA (IPEA). Biocombustíveis no Brasil: Etanol e Biodiesel. Rio de Janeiro: IPEA, 2010. (Eixos do Desenvolvimento Brasileiro, n. 53).

JANK, M. S. Etanol: Entendendo o Mercado e os Preços. O Estado de São Paulo, 22 de Jan. 2010. Disponível em: <http://www.unica.com.br/opiniao/show.asp?Msgcode=E7A1650F52BC-4BFC-866D-6C2BD9368776 > . Acesso em: 08 Jun. 2011.

MORETTIN, P. A.; TOLOI, C. M. Análise de Séries Temporais. São Paulo: Blucher, 2006.

NELSON, D. B. Conditional Heteroskedasticity in Asset Returns: A New Approach. Econometrica, Chicago, n. 59, n. 2, p.347-370, Mar. 1991.

NICOLA, D. S.; CLAILTON, A. F.; PAZ, M. V. Previsão dos Preços do Açúcar e Análise da sua Volatilidade no Mercado Futuro Brasileiro (2003 a 2007): Uma Aplicação de Modelos da Família ARCH. In: CONGRESSO DA SOCIEDADE BRASILEIRA DE ECONOMIA, ADMINISTRAÇÃO E SOCIOLOGIA RURAL, 46., 2008, Rio Branco. Anais... Rio Branco, 2008.

OÑATE, C. A. et al. Previsão das Variações de Preços do Etanol No Estado de São Paulo Usando Metodologias de Séries Temporais Univariadas. In: CONGRESSO DA SOCIEDADE BRASILEIRA DE ECONOMIA, ADMINISTRAÇÃO E SOCIOLOGIA RURAL, 48., 2010, Campo Grande. Anais... Campo Grande: SOBER, 2010.

PENA, D.; TIAO, G. C.; TSAY, R. S. A Course in Time Series Analysis. New York: John Wiley and Sons, 2001.

SERRA, T.; ZILBERMAN, D. Price Volatility in Ethanol Markets. In: AGRICULTURAL AND APPLIED ECONOMICS ASSOCIATION, 2009, Milwalkee. Annul Meeting ... Milwalkee, 2009.

SHIKIDA, P. F. et al. Uma Análise Econométrica Preliminar das Ofertas de Açúcar e Álcool Paranaenses. Revista de Economia Agrícola, São Paulo, n. 54, p. 21-32, 2007.

TADESSE, G.; GUTTORMSEN, A.G. The Behavior of Commodity Prices in Ethiopia. Agricultural Economics, Amsterdam, NL, v. 42, n. 2, p.87-97, 2011.

TEIXEIRA, G. A. et al. Dinâmica da Volatilidade do Retorno das Principais Commodities Brasileiras: Uma Abordagem dos Modelos ARCH. In: CONGRESSO DA SOCIEDADE BRASILEIRA DE ECONOMIA, ADMINISTRAÇÃO E SOCIOLOGIA RURAL, 46., 2009, Rio Branco. Anais... Rio Branco: SOBER, 2009.

TSAY, R. S. Analysis of financial time series. Hoboken: Wiley, 2005.

UNIÃO DA INDÚSTRIA DE CANA-DE-AÇÚCAR (ÚNICA). Dados e Cotações: Estatísticas: Vendas de automóveis e comerciais leves por tipo de combustível, 2011.

ZAKOIAN, J. M. Threshold Heteroskedasticity Models. Journal of Economic Dynamics and Control, Amsterdam, NL, v. 18, n. 5, p.931-955, 1994. 
Apêndice A - Informações Básicas e Teste de Normalidade

\begin{tabular}{cccc}
\hline Série & Assimetria & Curtose & Jarque Bera \\
\hline Preços & 0.9201 & 5.0849 & $\begin{array}{c}183.3583 \\
(0.0000) \\
\text { Retornos }\end{array}$ \\
& 0.8563 & 10.7104 & $\begin{array}{c}1479.035 \\
(0.0000)\end{array}$ \\
\hline
\end{tabular}

Nota: p-valor entre parênteses. Hipótese nula de normalidade.

Apêndice B - p-valor dos testes de Raiz Unitária

\begin{tabular}{ccc}
\hline Série & ADF & PP \\
\hline Preços & 0.0152 & 0.0495 \\
Retornos & 0.0000 & 0.0000 \\
\hline
\end{tabular}

Nota: hipótese nula de raiz unitária em ambos os testes.

Apêndice C - GARCH, EGARCH e TARCH para a série de retornos, 2000/2004

\begin{tabular}{cccc}
\hline Variância & GARCH (1,1) & EGARCH(1,1) & TARCH(1,1) \\
\hline$\alpha_{0}$ & $0.0000^{* * *}$ & $-2.4318^{* * *}$ & $0.0003^{* * *}$ \\
$\varepsilon_{t-1}^{2}$ & $(0.0040)$ & $(0.0000)$ & $(0.0000)$ \\
$\sigma_{t-1}^{2}$ & $1.8427^{* * *}$ & & 0.0225 \\
$d_{t-1} \varepsilon_{t-1}^{2}$ & $(0.0000)$ & $0.6150)$ \\
$\left|\varepsilon_{t-1}^{2}\right| /\left|\sigma_{t-1}\right|$ & $(0.0000)$ & & $(0.0000)$ \\
$\varepsilon_{t-1}^{2} / \sigma_{t-1}$ & & & $1.9724^{* * *}$ \\
$\ln \left(\sigma_{t-1}^{2}\right)$ & & $1.2045^{* * *}$ & $(0.0000)$ \\
$\mathrm{ARCH} \mathrm{LM} \mathrm{Test}$ & & $(0.0000)$ & \\
AIC & & $-0.4538^{* * *}$ & \\
SBC & 0.9946 & $0.0000)$ & \\
\hline
\end{tabular}

Fonte: Elaboração própria.

Nota: média condicional modelada por um modelo ARMA $(1,0)$. 
Apêndice D - GARCH, EGARCH e TARCH para a série de retornos, 2004/2009

\begin{tabular}{cccc}
\hline Variância & GARCH (1,1) & EGARCH(1,1) & TARCH(1,1) \\
\hline$\alpha_{0}$ & $0.0002^{* * *}$ & $-4.3529^{* * *}$ & $0.0002^{* * *}$ \\
$\varepsilon_{t-1}^{2}$ & $(0.0000)$ & $(0.0000)$ & $(0.0000)$ \\
$\sigma_{t-1}^{2}$ & $1.2268^{* * *}$ & & $(0.0000)$ \\
$d_{t-1} \varepsilon_{t-1}^{2}$ & $(0.0000)$ & & 0.0512 \\
$\left|\varepsilon_{t-1}^{2}\right| /\left|\sigma_{t-1}\right|$ & 0.0880 & & $(0.2044)$ \\
$\varepsilon_{t-1}^{2} / \sigma_{t-1}$ & $(0.1158)$ & & $-0.9277^{* *}$ \\
$\ln \left(\sigma_{t-1}^{2}\right)$ & & $1.0838^{* * *}$ & $(0.0117)$ \\
$\mathrm{ARCH} \mathrm{LM} \mathrm{Test}$ & & $(0.0000)$ & \\
AIC & & $0.1668^{* *}$ & \\
SBC & 0.4558 & $(0.0422)$ & \\
& -4.3458 & $(0.0000)$ & 0.2877 \\
\end{tabular}

Nota: média condicional modelada por um modelo ARMA $(2,2)$.

Apêndice E - GARCH, EGARCH e TARCH para a série de retornos, 2009/2011

\begin{tabular}{cccc}
\hline Variância & GARCH (1,1) & EGARCH(1,1) & TARCH(1,1) \\
\hline$\alpha_{0}$ & $0.0003^{* * *}$ & $-6.6722^{* * *}$ & $0.0003^{* * *}$ \\
$\varepsilon_{t-1}^{2}$ & $(0.0027)$ & $(0.0014)$ & $(0.0026)$ \\
$\sigma_{t-1}^{2}$ & $0.9651^{* * *}$ & & $\left(0.0055^{* * *}\right.$ \\
$d_{t-1} \varepsilon_{t-1}^{2}$ & $(0.0003)$ & & $0.2366^{* *}$ \\
$\left|\varepsilon_{t-1}^{2}\right| /\left|\sigma_{t-1}\right|$ & $0.2240^{* *}$ & $(0.0399)$ \\
$\varepsilon_{t-1}^{2} / \sigma_{t-1}$ & $(0.0460)$ & & -0.2512 \\
$\ln \left(\sigma_{t-1}^{2}\right)$ & & & $(0.5420)$ \\
$\mathrm{ARCH} \mathrm{LM} \mathrm{Test}$ & & $0.9361^{* * *}$ & \\
AIC & & $(0.0000)$ & \\
SBC & & -0.0005 & \\
& & $(0.9963)$ & \\
& & $0.6924^{* * *}$ & 0.1933 \\
& -3.5203 & $(0.0000)$ & -3.5053 \\
\end{tabular}

Nota: média condicional modelada por um modelo ARMA $(2,0)$.

Recebido em: 13/09/2011.

Aceito em: 20/03/2012. 